\section{Placing kinases in the WNT pathway}

\section{By Joanne Kotz, Senior Editor}

Despite the well-established role of WNT signaling in many cancers, the pathway has proven difficult to modulate therapeutically because it is largely composed of hard-to-target protein-protein interactions. Now, a Boston-based team and a group led by Roche's Genentech Inc. unit have each identified a new kinase regulator of WNT signaling-YES1 and RIPK4, respectively - that provide two new druggable targets in the pathway. ${ }^{1,2}$

Each team will now look for the cancer subtypes most likely to respond to inhibitors of these kinases.

The wingless-type MMTV integration site (WNT) pathway plays a critical role during development and in maintaining adult tissues. In the pathway, WNT ligands bind to frizzled receptors, triggering a signaling cascade that leads to $\beta$-catenin (CTNNB1) accumulating in the cytoplasm and then translocating to the nucleus, where it acts in transcriptional complexes to regulate gene expression (see Figure 1, "WNT signaling in cancer").

WNT pathway activation is likely a frequent driver of cancer. In colon cancer, somatic mutations that increase WNT signaling have been identified in multiple components of the pathway. In other cancer subtypes, WNT signaling is clearly elevated, but specific oncogenic alterations in the pathway have not been identified.

"The role of the WNT pathway has been very well established in colorectal cancer. Its relevance in other cancer types remains unclear," said Christoph Lengauer, CSO at cancer company Blueprint Medicines. "Irrespectively, in spite of many efforts by numerous companies and academic centers, drugging this pathway has been challenging. This is due to the complexity of WNT signaling and the lack of targets in this pathway that are accessible to drug discovery."

Few companies have taken WNT pathway modulators into the clinic in cancer. The most advanced program is OncoMed Pharmaceuticals Inc.'s vantictumab (OMP-18R5), an antibody that binds multiple frizzled receptors. The molecule, which is partnered with Bayer AG, started Phase I testing in solid tumors in 2011.

"Connecting kinases like YES1 and RIPK4 to the WNT pathway is exciting because kinases are druggable," Lengauer added.

\section{YES gets a nod}

A team led by William Hahn set out to identify new targets in $\beta$-catenin-driven cancers. ${ }^{1}$ Hahn is deputy CSO and chief of the Division of Molecular and Cellular Oncology at the Dana-Farber Cancer Institute and a senior associate member of the Broad Institute of MIT and Harvard.

First, the team looked at small hairpin RNA screening data to identify genes essential for proliferation or survival in a panel of cancer cell lines with increased levels of $\beta$-catenin activity. The screening data previously were generated through a joint effort by Dana-Farber and the Broad Institute called Project Achilles that included silencing more than 11,000 genes across about 100 genetically characterized cancer cell lines.

Surprisingly, transcription factor 4 (TCF4), which typically interacts with $\beta$-catenin to regulate gene expression, did not come up as essential.

This led the team to search for whether $\beta$-catenin could be forming a transcriptional complex that did not include TCF4 in these cancer cell lines.

In fact, many essential genes in the WNT-activated cancer cell lines were regulated instead by the transcriptional regulator yes-associated protein 1 (YAP1). Indeed, the team looked at colon cancer cell lines with activated WNT signaling and found that $\beta$-catenin formed a complex with YAP1, not TCF4, and this complex induced expression of antiapoptotic genes.

Because YAP1 originally was identified as a protein that interacts with v-yes-1 Yamaguchi sarcoma viral oncogene homolog 1 (YES1; Yes), the team next looked to see if YES1 could be regulating the activity of this transcriptional complex.

In a WNT-driven colon cancer cell line, YES1 interacted directly with YAP1, and knocking down YES1 decreased tumor formation in mice.

Finally, a panel of cancer cell lines with increased WNT activation was more sensitive to Sprycel dasatinib, an inhibitor of multiple kinases including YES1, than cancer cell lines without WNT pathway activation.

Sprycel is marketed by Bristol-Myers Squibb Co. to treat acute lymphoblastic leukemia (ALL) and chronic myelogenous leukemia (CML).

Results were published in Cell.

\section{RIP-ping into WNT}

A team led by Vishva Dixit, VP of physiological chemistry at Genentech, arrived at its new WNT target by a very different route. ${ }^{2}$

The group started from the observation that humans with mutations in the kinase receptor-interacting serine-threonine kinase 4 (RIPK4; RIP4) develop Bartsocas-Papas syndrome, a rare genetic disease characterized by severe developmental defects.

To look at the pathway regulated by RIPK4, the team overexpressed the target in human cells and saw changes in gene expression that were 
Figure 1. WNT signaling in cancer. A Boston-based team and a Genentech Inc.-led team have each identified a new kinase-v-yes-1 Yamaguchi sarcoma viral oncogene homolog 1 (YES1; Yes) and receptor-interacting serine-threonine kinase 4 (RIPK4; RIP4), respectively - that acts in wingless-type MMTV integration site (WNT)-driven cancers.

The Genentech team found that RIPK4 acts at the WNT receptor complex to phosphorylate dishevelled dsh homolog 2 (DVL2). This induces $\beta$-catenin (CTNNB1) accumulation in the cytoplasm (I[a]) and translocation to the nucleus to regulate gene expression via the TCF family of transcription factors (I[b]). RIPK4 also appears to play a role in cancer cells (II[a]), as the kinase is overexpressed in ovarian cancers, and knocking down RIPK4 in a cancer cell line decreased tumor growth in mice.

The Boston team found that in cancer cell lines with increased $\beta$-catenin activity, $\beta$-catenin forms a transcriptional complex with yes-associated protein 1 (YAP1) and YES1 that regulates the increased expression of antiapoptotic genes (II[b]). Knocking down YES1 decreased the formation of WNT-driven colon tumors in mice.

similar to those seen when treating the cells with a WNT ligand.

As a result, the team decided to probe whether the kinase might be regulating WNT signaling. To do so, the researchers first identified proteins that interacted directly with RIPK4, which led them to dishevelled dsh homolog 2 (DVL2), a component of the WNT receptor signaling complex. In cells, RIPK4 directly phosphorylated DVL2 in response to WNT pathway activation.

Because of the frequent role of WNT pathway activation in cancer, the team next asked if RIPK4 might be altered in the disease. Indeed, microarray data from human ovarian tumor tissue showed that RIPK4 was overexpressed compared with data from noncancerous ovarian tissue $\left(p=8.9 \times 10^{-25}\right)$.

Finally, the team looked to see if blocking RIPK4 might have a therapeutic effect. In a mouse xenograft model for a tumor driven by increased WNT receptor signaling, shRNA knockdown of RIPK4 almost completely suppressed tumor growth.

Results were published in Science.

\section{Picking patients}

A key next step will be determining the specific cancer patient subpopulations most likely to benefit from inhibiting RIPK4 or YES1.

"It is too early to predict which patient population will most benefit from RIPK4 inhibitors, but the data on RIPK4 overexpression in a large number of ovarian cancers together with its role regulating $\beta$-catenin levels makes a compelling case for targeting this protein,"

said Stefan Knapp, principal investigator at the Structural Genomics Consortium and director of chemical biology at the Target Discovery Institute in the Nuffield Department of Clinical Medicine at the University of Oxford.

"It is too early to predict
which patient population
will most benefit from RIPK4
inhibitors, but the data on
RIPK4 overexpression in
a large number of ovarian
cancers together with its role
regulating $\beta$-catenin levels
makes a compelling case for
targeting this protein."
-Stefan Knapp,
Structural Genomics Consortium

"The recent data on YES1 are thought provoking and exciting because they suggest a molecular connection between YAP1 and $\beta$-catenin. If the YAP1-YES1- $\beta$-catenin axis could be further substantiated, this would be truly exciting because YES1 is a kinase and potentially druggable," added Lengauer.

Knapp and Lengauer both said a logical next step would be developing selective kinase inhibitors that can be used in cancer cell lines and mouse models to further explore the potential of these kinases as therapeutic targets and identify potential biomarkers that predict response.

Lengauer said it is too early to tell how broad a role these kinases may play in WNT-driven cancers. "More tumors need to be studied because the role of $\beta$-catenin in cancerthough well established-is very complicated. Of course, it is unlikely that a single mechanism is important in all cancers of a particular subtype."

Indeed, both teams are now planning to look more closely at the role of these kinases in different cancer subpopulations.

Hahn told SciBX that the Boston team is setting up screens to look for YES1 or YAP1 inhibitors. With inhibitors in hand, the researchers plan to test whether specific inhibition of these targets recapitulates 


\section{ANALYSIS}

the genetic findings.

The Genentech team is now "carefully evaluating the potential of RIPK4 as a cancer target," said XiaoDong Andy Huang, a scientist in the Department of Molecular Diagnostics and Cancer Cell Biology at Genentech and the first author on the paper.

Huang cautioned that because RIPK4 sits far upstream in the WNT pathway, it is unlikely to have a therapeutic effect in many cancers with activated WNT signaling. He noted that most $\beta$-cateninactivated tumors are due to mutations of adenomatous polyposis coli (APC) (about $85 \%$ ) or $\beta$-catenin itself (about $10 \%$ ). APC is a negative regulator of $\beta$-catenin stability in the cytoplasm.

"Therefore, a RIPK4 inhibitor is unlikely to have efficacy against those tumors since RIPK4 functions at an upstream level," said Huang. "Most likely, RIPK4 inhibitors will have an effect on tumors with abnormally high WNT pathway activity caused by players at or upstream of RIPK4, for example, WNT ligand overexpression or chromosome translocations of R-Spondin family members."

In 2012, a Genentech team reported in Nature that about $10 \%$ of tumors from patients with colon cancer contain gene fusions of $\mathrm{R}$-Spondin family members $R$-Spondin 2 (RSPO2) or RSPO3. Those genes code for secreted proteins known to potentiate WNT signaling, and the genetic alteration led to aberrant activation of the WNT pathway. ${ }^{3}$

A patent application has been filed by Dana-Farber and the Broad
Institute covering the work reported in Cell. The IP is available for licensing

Genentech did not disclose the patent or licensing status of its work.

Kotz, J. SciBX 6(10); doi:10.1038/scibx.2013.231

Published online March 14, 2013

\section{REFERENCES}

1. Rosenbluh, J. et al. Cell; published online Dec. 13, 2012; doi:10.1016/j.cell.2012.11.026

Contact: William C. Hahn, Dana-Farber Cancer Institute, Boston, Mass.

e-mail: william_hahn@dfci.harvard.edu

2. Huang, X. et al. Science; published online Jan. 31, 2013; doi:10.1126/science.1232253

Contact: Vishva M. Dixit, Genentech Inc., South San Francisco, Calif. e-mail: dixit@gene.com

3. Seshagiri, S. et al. Nature 488, 660-664 (2012)

COMPANIES AND INSTITUTIONS MENTIONED

Bayer AG (Xetra:BAYN), Leverkusen, Germany

Blueprint Medicines, Cambridge, Mass.

Bristol-Myers Squibb Co. (NYSE:BMY), New York, N.Y.

Broad Institute of MIT and Harvard, Boston, Mass.

Dana-Farber Cancer Institute, Boston, Mass.

Genentech Inc., South San Francisco, Calif.

OncoMed Pharmaceuticals Inc., Redwood City, Calif.

Roche (SIX:ROG; OTCQX:RHHBY), Basel, Switzerland

Structural Genomics Consortium, Oxford, U.K.

University of Oxford, Oxford, U.K. 